\title{
Neuer „Hofadel“ am Seerheinufer Konstanz? \\ Machteffekte im restrukturierten Raum
}

\section{Einleitung}

Der folgende Text beschäftigt sich mit dem Zusammenhang von Macht und Raum im Zuge der aktuellen städtebaulichen Maßnahmen am Konstanzer Seerheinufer. Der Seerhein ist die Verengung des Bodensees, der an dieser Stelle zu einem Fluss wird, wobei Konstanz auf beiden Seiten des Seerheins liegt. Auf einem ehemaligen Fabrikgelände gegenüber der Konstanzer Altstadt ist seit dessen Bebauung 2004 ein neues, großzügig gestaltetes Wohnquartier und ein großflächig angelegtes öffentliches Parkgelände entstanden. Um die Nutzung der angrenzenden Uferpromenade wird seit Jahren gestritten. Zur Lösung des Konflikts beschäftigen die neuen Bewohner_innen des Quartiers selbst einen privaten Sicherheitsdienst, der zusätzlich zur Polizei für Sicherheit und Ordnung sorgen soll. Dass dieser gleichzeitig im öffentlichen Park, zeitweise auch zur Durchsetzung des städtischen Glasverbots, patrouilliert, zeigt beispielhaft die Etablierung umfassender Kontrollpolitiken.

Neoliberale Sicherheitspolitiken entstehen zwischen unterschiedlichen Akteuren, wobei zunehmend privatwirtschaftliche und zivilgesellschaftliche Akteure mit einbezogen werden (Kern 2014: 17). Zum einen ist deshalb zu fragen, welche Akteure an den räumlich begrenzten Sicherheitspolitiken beteiligt sind und welche Diskurse und Praktiken bei der Konstruktion des Raumes als ,Brennpunkt‘ im untersuchten Fallbeispiel zum Tragen kommen. Zum anderen ist zu fragen, inwiefern sich die Produktion von Sicherheit in Bezug auf den restrukturierten Raum als Mittel zur strategischen Nutzung des Raumes begreifen lassen, durch dessen herrschaftsförmige Gestaltung sich gesellschaftliche Verhältnisse reproduzieren.

Nach einer kurzen Vorstellung des Fallbeispiels wird die Konstruktion des ehemaligen Fabrikgeländes als, Gefahrengebiet ' in diesem Beitrag in den Kontext einer geographisch-historischen Raumanalyse gestellt, wie sie Bernd Belina (2013) vorschlägt. Indem die am Seerheinufer etablierten Sicherheitsund Kontrollpolitiken als „Territorialisierungsprozesse“ (ebd.: 88 ff.) verstanden werden, wird der Beitrag diese räumlich bezogene soziale Praxis jenseits einer Reduzierung und Abstraktion auf räumlich bedingte kulturelle 
Praktiken und Diskurse auf der Grundlage empirischer Daten analysieren. Dazu wird das ausdifferenzierte Akteursfeld skizziert, das sich im Fallbeispiel an den erweiterten Maßnahmen der Sicherheitsproduktion beteiligt. Die unterschiedlichen Strategien und Interessen der verschiedenen Akteure und Institutionen bezüglich der Sicherheit im neuen Stadtteilgebiet werden hierbei in ihrem spezifischen historischen und gesellschaftlichen Kontext betrachtet.

Letztendlich stellt der Text die Frage, welche Anregungen aus dem Fallbeispiel für eine machtkritische Analyse neoliberaler Sicherheitsregime innerhalb urbaner Raumentwicklung insgesamt gewonnen werden können. Das Thema und zugleich der Kontext dieses Beitrags bieten somit auch die Möglichkeiten einer Analyse und Kritik der herrschaftsförmigen Gestaltung des urbanen Raumes im Allgemeinen.

\section{Fallstudie zum ,Gefahrengebiet' ${ }^{\text {in Konstanz }}$}

Das Konstanzer Seerheinufer steht seit einigen Jahren im Fokus der städtischen Bebauungsplanung. Seit der Produktionseinstellung der Textilfabrik Herosé 2001 ist dort Wohnraum mit etwa 300 neuen Wohnungen entstanden. Zusätzlich wurden modern entworfene und repräsentative Bürogebäude entlang des attraktiven Uferbereichs angesiedelt. Politisch-städtebauliche Maßnahmen führten so zu einer Neustrukturierung des alten Fabrikgeländes in einen neuen „Wohn-, Büro- und Dienstleistungsstandort“ (Pressereferent der Stadt Konstanz o. J.), der außerdem mit dem großzügigen, neu angelegten Herosé-Park und einer nun öffentlich zugänglichen Uferpromenade eine ökonomische Aufwertung des gesamten gegenüber der Konstanzer Altstadt gelegenen Uferbereichs am Rhein darstellt.

Anschließend an den Herosé-Park entstand hier ein neues Wohnquartier. Es besteht aus mehreren zum Rhein hin offenen Wohnanlagen, die, jeweils hufeisenförmig gebaut, durch eine zum Ufer des Seerheins hin offene Garten- bzw. Parkanlage[1] zur privaten Nutzung durch die Bewohner_innen abgegrenzt werden (vgl. Abb. 1). Dies führte in Folge zu einigen Interessenskonflikten zwischen den neuen Anwohnern_innen und Eigentümer_innen im Quartier einerseits und den Nutzern_innen der öffentlichen Promenade und des anschließenden Parks andererseits. Dabei organisieren sich einige der Anwohner_innen des neuen Stadtquartiers als zivilgesellschaftliche Interessengemeinschaft und unterhalten gemeinsam die Homepage (Seerhein-Netz)[2], um ihre Interessen gegenüber der Stadtverwaltung und dem Stadtrat geltend zu machen. Als Probleme, die durch die freie Nutzung der anschließenden Parkanlage

Abb. 1 Bebauungsplan Konstanzer Seerheinufer (Quelle: Klaus Theo Brenner)

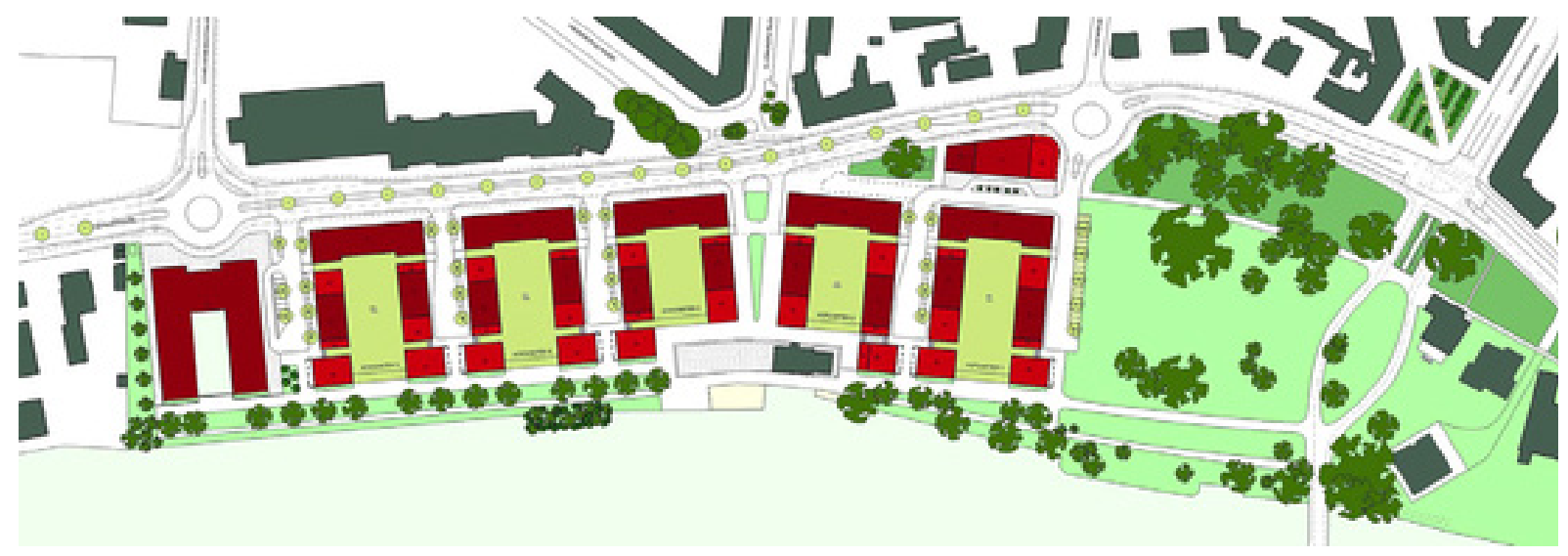



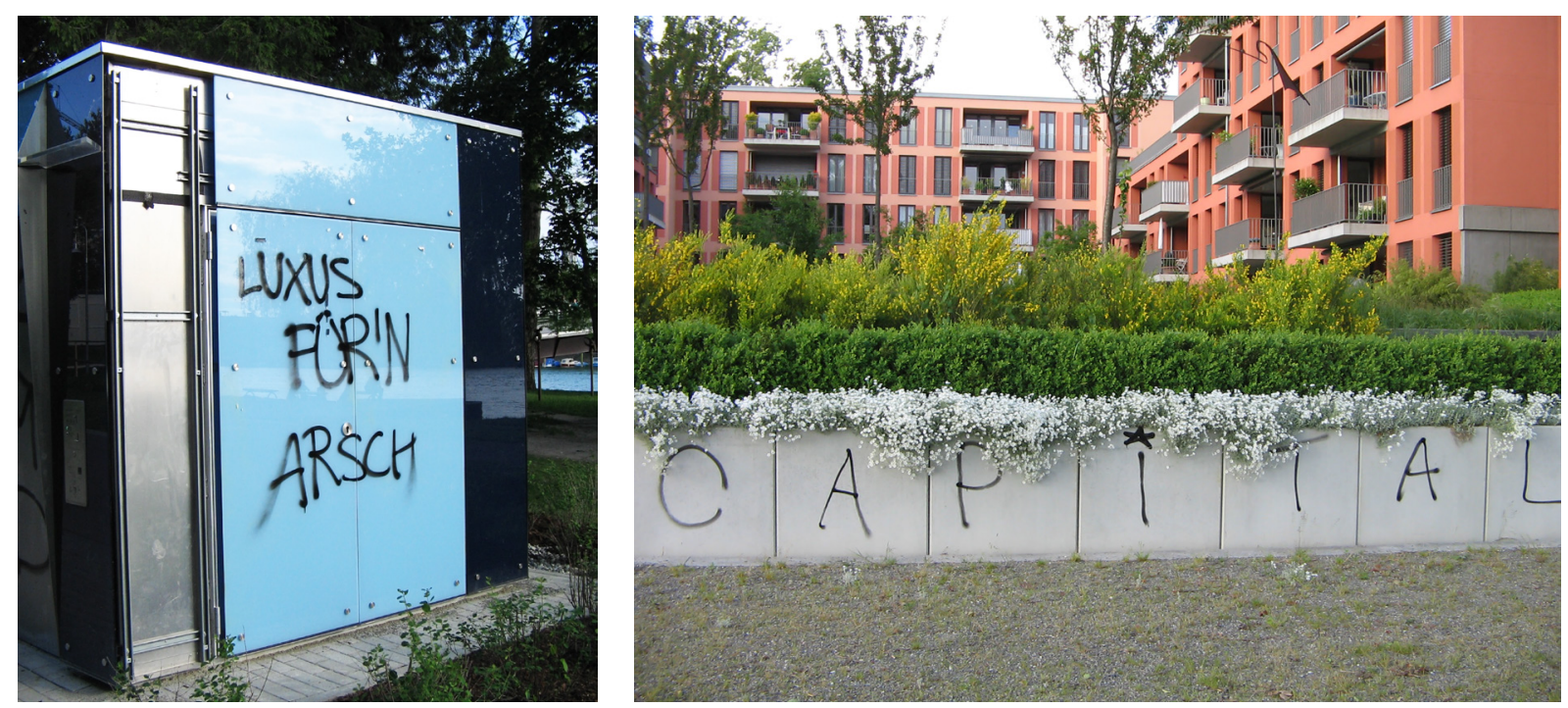

Abb. 2 Beschädigung der gebührenpflichtigen Toilette im Park (Quelle: Anja Joos)

Abb. 3 Graffiti gegen teuren Wohnraum (Quelle: Anja Joos) entstünden, betrachten sie „Lärm, Müll, Belästigung von Passanten“(SeerheinNetz o. J.) sowie Beschädigungen im Quartier. Nachdem die Polizei jedoch aufgrund der eher geringen Kriminalitätsbelastung keinen erhöhten ordnungspolitischen Bedarf erkennen konnte (vgl. Pugliese 2014), setzten die Eigentümer_innen und Mieter_innen ihre Forderungen an die Stadt, gegen Müll und Lärmbelästigung vorzugehen, selbst durch. In diesem Konflikt um den neuen öffentlichen Raum haben die Bewohner_innen des Quartiers daher über mehrere Jahre einen kommerziellen Sicherheitsdienst engagiert und finanziert, der auf dem Gelände des öffentlichen Parks und entlang der öffentlichen Promenade zum Einsatz kam.

Obwohl eine öffentliche Finanzierung des privaten Sicherheitsdienstes in Briefen und Unterschriftenaktionen von engagierten Anwohner_innen wiederholt gefordert worden war, lehnte der Gemeinderat (Stadt Konstanz 2014a) dies im Sommer 2014 zwar erneut ab (vgl. Seerhein-Netz 2011). In der historischen Betrachtung zeigt sich allerdings, wie diese Initiative - quasi als Interessenvertretung des Quartiers - teilweise zusammen mit der ,Bürgergemeinschaft Petershausen'[3], entsprechend Einfluss auf stadtpolitische Entscheidungen ausübte, um die Produktion des öffentlichen Raumes ihren Interessen entsprechend auszurichten. Auf die mehrmalige Bitte der zivilgesellschaftlichen Initiative hin wurde bereits 2011 ein Glasverbot von der Stadtverwaltung eingeführt (vgl. Seerhein-Netz o. J.). Bevor gegen diese Sondergesetzgebung 2012 erfolgreich Klage beim Verwaltungsgericht Mannheim eingelegt wurde, diente der privat organisierte Sicherheitsdienst über zwei Jahre hinweg vor allem dazu, diese ordnungspolitische Maßnahme auf dem öffentlichen Gelände durchzusetzen und zu gewährleisten.

\subsection{Diskursive Strategien}

In der diskursiven Legitimierung solcher macht- und ordnungspolitischen Maßnahmen durch die zivilgesellschaftlich engagierten Anwohner_innen tritt das neustrukturierte Areal vor allem als „Brennpunkt“ (SeerheinNetz o. J.) in den Vordergrund. Dies lässt sich empirisch besonders anhand des zahlreich vorliegenden Materials auf der Homepage herausarbeiten, auf der deren Autor_innen in seriell dokumentierten Erfahrungs- und Erlebnisberichten zu einzelnen Bagatelldelikten[4] auf ihr Leid verweisen. In diesen 
Berichten werden die neuen Bewohner_innen des Quartiers dichotom anderen Nutzergruppen des öffentlichen Geländes gegenübergestellt, die dabei fast ausschließlich stereotypisierend als ,Chaoten', ,Volltrunkene', ,die Störenden' und als ,Vandalierer' (vgl. Seerhein-Netz o. J.) kategorisiert werden. Das auf diese Weise konstruierte Wissen und dessen Machtwirkungen werden von der lokalen Tageszeitung Südkurier reproduziert, sodass sich eben jene Diskurseffekte verstärken, die die Vorstellung des Raumes nach den Kriterien von Sicherheit und Ordnung ausrichten.

Darauf, dass in medialen Berichten des Südkuriers wenig zwischen unterschiedlichen Nutzer_innen differenziert werde und damit deren Stereotypisierung vorangetrieben werde, weist auch die Studie der Mediatorin Franziska Becker hin (2013: 9), die in diesem Konflikt von der Stadt engagiert wurde. Sie geht davon aus, dass die geringen Erfolgsaussichten von teils dialogorientierten Verfahren im untersuchten Quartier vor allem in der fehlenden Bereitschaft der Eigentümer_innen und Mieter_innen begründet sind, vermittelnde Gespräche mit den Nutzer_innen der öffentlichen Parkanlage zu führen und sich auf „ergebnisoffene Verfahren einzulassen“ (ebd.: 21). Vor allem die engagierten Autor_innen der Homepage lehnen ,pädagogische“ Maßnahmen ab und fordern für das Seerheinufer restriktive Verbote und Kontrollen. Wie eine weitere Studie zeigt, lehnt dagegen das junge und studentisch geprägte Milieu der Nutzer_innen restriktive Maßnahmen wie das Glasflaschenverbot ab (Edinger/Lipphardt 2011: 10); 70 Prozent der Anwohner_innen konstatieren überhaupt kein problematisches Nutzungsverhalten (ebd.: 7). Trotzdem werden über die wenig differenzierende Berichterstattung in der Lokalzeitung die Partikularinteressen einiger engagierter Anwohner_innen in die Öffentlichkeit getragen.

Diese Interessen werden in der Folge auch durch stadtpolitische Akteure geteilt. Besonders die Stadtverwaltung zeigt sich unzufrieden über die „unbefriedigende, aber aktuelle Rechtslage“ (vgl. Stadt Konstanz 2014c), die keine Regelungen über das bestehende Recht zum Lärmschutz oder die geltende Umwelt- und Polizeiverordnung hinaus vorsehe, um „den Anwohnern in ihrem Recht auf Nachtruhe zu verhelfen“ (ebd.: 2014).

\subsection{Lokalstaatliche Interventionen}

Die Verschränkung der Interessen zur gemeinsamen Herstellung von Sicherheit im neustrukturierten Raum kommt neben dem zeitweise gültigen Glasverbot in anderen, weitreichenden Forderungen lokalstaatlicher Akteure nach Maßnahmen zur Überwachung und Kontrolle des öffentlichen Geländes zum Ausdruck. Sie fordern „eine Videoüberwachung zu installieren“ (Stadt Konstanz 2014b) oder „einzelne Plätze an der Bischofsvilla[5] für den Aufenthalt uninteressanter zu machen“ (Rindt 2014), indem diese entsprechend bebaut werden. Hiermit tritt die lokalstaatliche Politik selbst als strategisch und taktisch handelnder Akteur in den Vordergrund.

Anhand des empirischen Materials zeigt sich, dass stadtpolitische Akteure durch vielfältige Strategien, die sich bis in die sozialpädagogischen Diskurse und Maßnahmen hinein spiegeln, die Kontrolle im urbanen Raum über die Verfolgung von Kriminalität hinaus legitimieren und mit hervorbringen. Die neoliberale Sicherheitsproduktion bringt neben 
Abb. 4 Neugestalteter Uferbereich gegenüber der Konstanzer Altstadt (Quelle: Anja Joos)

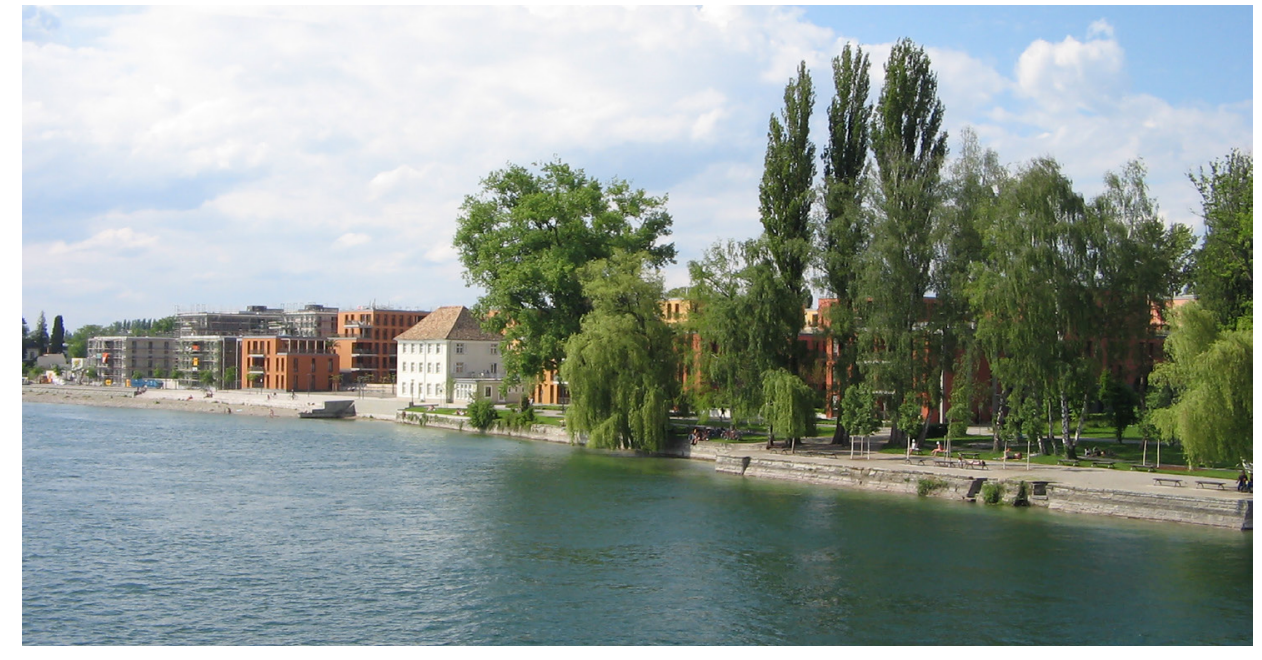

den bereits genannten restriktiven Maßnahmen am Konstanzer Ufer auch präventive Maßnahmen und ein weites Netz einer ,weichen' sozialen Kontrolle hervor. Dies zeigt sich in der städtischen Zusammenarbeit mit sozialpädagogischen Einrichtungen der Jugendarbeit, der Einrichtung eines Runden Tisches (vgl. Seerhein-Netz o. J.), der Prüfung von Mediations-Verfahren (vgl. Becker 2013) sowie auch in der Einrichtung einer Stelle zur Kommunalen Kriminalitätsprävention (KKP) (vgl. Stadt Konstanz 2014c). Zusammengenommen stellen all diese Maßnahmen Versuche der Stadtverwaltung und des Gemeinderates dar, einen erweiterten Handlungsspielraum „zur Verbesserung der soziale Kontrolle“ (Stadt Konstanz 2014c) im ökonomisch aufgewerteten Areal zu errichten. Die genannten, zum Teil restriktiven Strategien sollen solche Praktiken in dem Areal verhindern, die sich nicht auf „touristisches Schlendern“ (vgl. Stadt Konstanz 2014c) beschränken und somit nicht der Steigerung des Standortfaktors der „unternehmerischen Stadt“ (Harvey 1989) Konstanz dienen.

Insgesamt lässt sich konstatieren, dass in der Verschränkung zivilgesellschaftlich organisierter Akteure und lokalstaatlicher Politik verschiedene Taktiken und Strategien zur „(De-)Normalisierung“ (vgl. Link 1997)[6] der Nutzer_innen und deren Verhalten vorangetrieben werden, die die Verlängerung sozialer Kontrolle durch den privaten Sicherheitsdienst im öffentlichen Raum bis in die Subjekte hinein ermöglichen. Dazu erhält das öffentliche Parkgelände im Zusammenwirken privater, zivilgesellschaftlicher und staatlicher Akteure einen privaten Status, obwohl es gleichzeitig weiterhin als öffentlicher Raum funktioniert (Ronneberger 1999: 53). Mit dem so geschaffenen, „semi-öffentlichen“(Wehrheim 2012: 62) Raum werden auch Voraussetzungen für präventive Kontrollmaßnahmen über die bestehenden Gesetzesgrundlagen hinaus geschaffen, die dazu dienen, die Aufwertung des ehemaligen Fabrikgeländes sowie dessen marktförmige Gestaltung als neuer „Wohn-, Büro- und Dienstleistungsstandort“ (Pressereferent der Stadt Konstanz o. J.) durchzusetzen. In der skizzierten „historisch-spezifischen Konfiguration staatlicher und gesellschaftlicher Kontrollpolitiken“ (Kern 2014: 22) offenbaren sich Strategien, mit denen das Versprechen der städtischen Wohnungsbaupolitik, im restrukturierten Raum ein ruhiges und sauberes Umfeld in entsprechender Lage zu schaffen, auch von dem sozialstrukturell privilegierten Klientel selbst umgesetzt und aufrechterhalten werden soll (vgl. BHS Städtebau 2005). 


\section{Zur räumlich begrenzten Produktion von Sicherheit als Strategie}

Die Inanspruchnahme eines bestimmten abgegrenzten Gebiets durch soziale Praxen und Prozesse versteht Belina als „Territorialisierung“ (2013: 57 ff.). Über entsprechende Territorien wird insofern Macht ausgeübt, als die raumbezogene soziale Praxis und die entsprechenden jeweiligen Prozesse nie allein einem Selbstzweck dienen, sondern je nach Interesse und ihrem Gegenstand nach als Strategien fungieren. Dem geographisch-historischen Materialismus Belinas folgend, sind Produktion und Aneignung physischmaterieller Räume „nicht Ergebnis individueller Praxis, sondern Momente gesellschaftlicher Verhältnisse“ (ebd.: 50). Dem entgegenstehend werden nach dem cultural turn der Sozialwissenschaften Räume vor allem als kulturell geprägt und sozial konstruiert begriffen. Damit erscheinen Räume und ihre Machtstrukturen stets als diskursiv hergestellt, so dass ihre physische Materialität selbst unberücksichtigt bleibt und sie letztlich nur als eine Idee erscheinen, quasi nur als eine Abstraktion, deren Resultat sie sind. Um nicht einseitig dem Idealismus ,kulturalisierter Räume` zu verfallen, scheint es daher wichtig, Territorialisierungsprozesse in ihrer Möglichkeit und Realität aus sozialen Prozessen heraus zu verstehen. Damit lässt sich die Konstruktion von Sicherheit und Ordnung nicht nur als Machteffekt im Raum verstehen, sondern es lässt sich darüber hinaus untersuchen, wie dieser als strategisches Mittel zum Zweck der Reproduktion gesellschaftlicher Verhältnisse eingesetzt wird. Betrachtet man entsprechend Sicherheitspolitiken als Strategien der Territorialisierung in Bezug auf den restrukturierten Raum, lassen sich auch „Spuren des gesellschaftlichen Charakters der Stadterneuerung aufspüren“ (Holm 2006: 11).

Zwar zeigt sich im untersuchten Fallbeispiel, wie im Zuge räumlich beschränkter Überwachungs- und Kontrollmaßnahmen unterschiedliche Akteure und Institutionen den städtischen Raum diskursiv herstellen, indem sie diesem direkt Bedeutungen zuweisen. Dabei entstehen entlang spezifischer Bedeutungszuschreibungen „dominante Vorstellungen“(Belina 2013:57 ff.) über die Sicherheit im Raum. Wie die physisch-materiellen Räume und eine auf diese bezogene soziale Praxis selbst gehen jedoch auch deren Bedeutungen aus gesellschaftlich umkämpften Prozessen hervor (ebd.: 55). Um dabei einer historisch-materialistischen Perspektive gerecht zu werden, können daher nicht nur „menschliche Bedeutungszuschreibungen (Wissen) für die Konstitution des städtischen Areals und/oder menschlichen Handelns in den Vordergrund gestellt“ (Christmann/Mahnken 2012: 91) werden.

Genauso wie die Sicherheitspraktiken stellt der hier vorgeschlagene Ansatz auch die Diskurse über die Sicherheit in den Kontext der materiellen Voraussetzungen, die zum Beispiel mit der Aufwertung eines Stadtteils als Teil gesellschaftlicher Prozesse in Verbindung stehen. Deshalb werden ,insbesondere die rechtlichen und ökonomischen Rahmenbedingungen, aber auch die administrativen Arrangements des lokal-staatlichen Handelns" (Holm 2006: 11) als wesentliche Ausgangslage der Forschung betrachtet. Dabei treten Lokalisierung und Regionalisierung als Kennzeichen neoliberaler Sicherheitsproduktion, um abgegrenzte urbane Räume als Ressource in der Standortpolitik nutzbar zu machen, in den Vordergrund der Analyse. (Kern 2014: 17). Jan Kemper und Anne 
Vogelpohl (2013) legen in ihrem programmatischen Aufsatz „Zur Konzeption kritischer Stadtforschung" dar, dass es um

„...die Berücksichtigung der historisch-sozialen Voraussetzungen für gegenwärtige Stadtentwicklungsprozesse, die Analyse der städtischen Raum- und Sozialverhältnisse als krisen- und konfliktvermittelte und deshalb als dynamische Verhältnisse sowie die Konzeptionierung dieser Verhältnisse als veränderbar [geht]“ (ebd.: 7).

Vor diesem Hintergrund kann die Bedeutung eben dieser „Verräumlichung von Sicherheit“" (Belina 2011) durch unterschiedliche Akteure angemessen analysiert und als gesellschaftlicher Prozess nachvollzogen werden.

\section{Fazit}

Die Analyse zeigt, wie im Zusammenwirken privater, kommerzieller und staatlicher Interessen und Strategien die eigentlich polizeiliche Aufgabe, für Sicherheit und Ordnung zu sorgen, auf verschiedene Akteure ausgelagert wird und sich die Kontrolle zusätzlich auf präventive Maßnahmen ausdehnt. Bei der gemeinsamen Produktion des Raumes bringen eine zivilgesellschaftliche Initiative und städtisch-administrative Institutionen in ihrer Verzahnung außerdem dominante Vorstellungen hervor, die in ihren konkreten Maßnahmen Relevanz für die soziale Praxis erlangen: Potenziell verstößt somit jede offene Weinflasche innerhalb des betrachteten Areals gegen bestimmte Normvorstellungen. Die diskursive Konstruktion des Raumes als ,Brennpunkt‘ dient der Legitimierung macht- und ordnungspolitischer Maßnahmen. Mittels dieses semiotisch vereinfachten Wissens bleiben andere Perspektiven auf den öffentlichen Raum, wie sie sowohl von den unterschiedlichen Nutzer_innen als auch von den Anwohner_innen selbst vertreten werden, unberücksichtigt. Die dominanten Vorstellungen in Bezug auf den öffentlichen Raum werden dabei in der lokalen Zeitung verstärkt und reproduziert. Somit wird der öffentliche Raum am Ufer des Seerheins auch im medialen Diskurs nach den Maßstäben von Sicherheit und Ordnung konstruiert.

Zwarlässt sich einerseits in diesen dominanten Vorstellungen und Praktiken zur Produktion von Sicherheit und Ordnung die „(De-)Normalisierung“bestimmter Verhaltensweisen im Raum erkennen. Andererseits scheint es wichtig, dass die spezifischen Formen der Subjektivierung durch individualisierte Kontrollmechanismen nicht isoliert betrachtet werden, wodurch sozialräumliche Prozesse als rein ideell und abstrakt produzierte Wirklichkeit erscheinen, in deren Folge das Sicherheitsregime nur als kulturelles Muster analysierbar wird. Hingegen ist es aus einer historisch-materialistischen Perspektive wichtig zu zeigen, dass im Zusammenwirken der verschiedenen Akteure zur Herstellung eines ruhigen und sauberen Umfeldes und der Ausrichtung des Raumes am Ideal des ,touristischen Schlenderns' dieser Raum zugleich als strategische Ressource zum Einsatz kommt und als Mittel fungiert, um das aufgewertete Fabrikgelände herrschaftsförmig zu organisieren. Dies bedeutet vor allem, dass die abstrakte Idee von Sicherheit im Raum sich nicht als Eigenlogik entfaltet, sondern innerhalb der konfliktiven Auseinandersetzung um Sozial- und Raumverhältnisse als Teil von Territorialisierungsstrategien zu begreifen ist. 
Die Produktion des Raumes lässt sich so als sozialer Prozess erkennen, der von den Strategien und Interessen einzelner Akteure bestimmt ist. Die Analyse macht die materiellen Bedingungen der Aufwertung sichtbar. Die ökonomische Aufwertung selbst als Machtstrategie im Raum zu betrachten, macht diese als einen Teil der kapitalistischen Verwertungslogik erkennbar, die den Raum zur strategischen Ressource innerhalb gesellschaftlicher Herrschaftslogik insgesamt macht. Eine rein diskursive Analyse, die die dominante Vorstellung von Sicherheit im Raum insgesamt in ihrem Verhältnis zur ökonomischen Aufwertung der ,Stadt am Wasser" ausblendet, würde somit der gleichen ideellen Konstruktion von Sicherheit und Ordnung im Raum folgen, mit der die engagierten Bewohner_innen des neuen Quartiers dem Raum seinen abstrakten sozialen Inhalt zuweisen.

Gleichzeitig ist für eine Analyse neoliberaler Kontrollpolitiken wichtig, dass sich Raum und Gesellschaft auch aus historisch-materialistischer Perspektive gegenseitig bedingen und Räume nicht lediglich als Spiegel der ökonomischen und politischen Verhältnisse zu begreifen sind. Versteht man vielmehr Raum als Elaborat gesellschaftlicher Verhältnisse und zugleich als Instrument, welches diese erst hervorbringt und stabilisiert, lässt sich der doppelte Zusammenhang von gesellschaftlichen Voraussetzungen und Effekten der Stadterneuerung zeigen.

\section{Endnoten}

[1] Diese Wohnquartiere werden daher im lokalen Sprachgebrauch aufgrund ihrer Bauweise auch ,Hofgärten' genannt.

[2] Leider lassen sich die Autoren der Homepage zumeist nicht direkt zuweisen, da die einzelnen Beiträge oft ohne kenntliche(n) Autor_in und Datum veröffentlicht sind. Ich habe mich deshalb darauf festgelegt, die Abkürzung „Seerhein-Netz“ zu benutzen und wenn möglich das Datum anzugeben. Als Mitautor_innen werden auf der Seite neben dem Betreiber (Günther Lange) noch B. Lange, H.-P. Krause und S. Geiger gelistet. Die Homepage (www. am-seerhein.net) selbst ist mittlerweile nur noch über archive.org aufrufbar.

[3] Petershausen ist der Konstanzer Stadtteil, dem auch das alte Fabrikareal angehört. Die Bürgergemeinschaft ist eine Stadtteilinitiative.

[4] Die Beschwerden beziehen sich dabei beispielsweise auf ein beschädigtes Toilettenhaus im Park (vgl. Abb. 2), einige rausgerissene Weidenstecklinge und wenige Graffitis (vgl. Abb. 3), die als politische Äußerung gegen den aufgewerteten Wohnraum verstanden werden müssen (vgl. Seerhein-Netz o.J.).

[5] Dies ist ein altes Gebäude, dessen Räumlichkeiten bereits vor der Restrukturierung des Fabrikgeländes der Universität Konstanz zur Verfügung standen.

[6] Interessant ist hierbei auch, dass Link (1997: 77) „normalistische Strategien“ besonders hervorhebt, die „im Sinne einer gerichteten Kombination einzelner ,Taktiken““ zu begreifen sind.

\section{Autor_innen}

Der Autor betreibt kritische Sozialforschung und bezieht dazu Anregungen aus der Sozial- und Kulturtheorie sowie der Ethnologie mit ein. Besonders die Themen Popkultur, Stadt- und Migrationsforschung stehen dabei im Fokus.

linus.klappenberger@hu-berlin.de 


\section{Literatur}

Becker, Franziska (2013): Nachtruhe versus Party? Eine ethnographische Studie zu Konflikten im öffentlichen Raum in Konstanz. Abschlussbericht der ethnographischen Orientierungsstudie (Prämediation) zu Konflikten im öffentlichen Raum Konstanz. http:// www.ethnologie-mediation.de/sicherheit-pravention (letzter Zugriff am 29.03.2016).

Belina, Bernd (2011): Raum, Überwachung, Kontrolle. Vom staatlichen Zugriff auf städtische Bevölkerung. Münster: Westfälisches Dampfboot.

Belina, Bernd (2013): Raum. Zu den Grundlagen eines historisch-geographischen Materialismus. Münster: Westfälisches Dampfboot.

Christmann, Gabriela B. / Mahnken, Gerhard (2012): Raumpioniere, stadtteilbezogene Diskurse und Raumentwicklung. Über kommunikative und diskursive Raum(re)konstruktionen. In: Rainer Keller / Inga Truschkat (Hg.): Methodologie und Praxis der wissenssoziologischen Diskursanalyse. Wiesbaden: VS Verlag.

Edinger, Eva / Lipphardt, Anna (2011): Melting Space Herosé? - Die „Stadt am Seerhein“ in Konstanz aus raumwissenschaftlicher Perspektive. http://kops.uni-konstanz.de/ handle/123456789/12656 (letzter Zugriff am 29.03.2016).

Harvey, David (1989): From managerialism to entrepreneurialism. The transformation in Urban Governance in late capitalism. In: Geografiska Annaler B 71, 3-17.

Holm, Andrej (2006): Die Restrukturierung des Raumes. Stadterneuerung der 9oer Jahre in Ostberlin: Interessen und Machtverhältnisse. Bielefeld: transcript Verlag.

Kemper, Jan / Vogelpohl, Anne (2013): Zur Konzeption kritischer Stadtforschung. Ansätze jenseits einer Eigenlogik der Städte. In: $\mathrm{s} \mathrm{u} b \backslash \mathrm{u}$ r b a n. Zeitschrift für kritische Stadtforschung 1/1, 7-30. http://www.zeitschrift-suburban.de/sys/index.php/suburban/article/ view/2/102 (letzter Zugriff am 29.03.2016).

Kern, Anna (2014): Ein Frankfurter Sicherheitsregime. Neoliberale Sicherheitsproduktion in der ,Hauptstadt des Verbrechens‘. In: $\mathrm{s} \mathrm{u} \mathrm{b} \backslash \mathrm{u}$ r b a n. Zeitschrift für kritische Stadtforschung 2/2, 17-38. http://www.zeitschrift-suburban.de/sys/index.php/suburban/ article/view/133/206 (letzter Zugriff am 29.03.2016).

Link, Jürgen (1997): Versuch über den Normalismus. Wie Normalität produziert wird. Opladen: Westdeutscher Verlag.

Pressereferent der Stadt Konstanz (o.J.): Stadt am Seerhein. Arbeiten und Wohnen am Wasser. http://www.konstanz.de/umwelt/01029/02010/05903/05940/index. html?lang=de (letzter Zugriff am 29.03.2016).

Pugliese, O. (2014): Bürger auf den Barrikaden. In: Seemoz, 20.10.2014.

Rindt, Claudia (2014): Patt bei Thema Sicherheitsdienst für das Seeufer. In: Südkurier, 12.02.2014.

Ronneberger, Klaus / Lanz, Stephan / Jahn, Wehrheim (Hg.) (1999): Die Stadt als Beute. Bonn: Dietz.

Seerhein-Netz (2009): Homepage der Bewohner der Stadt am Seerhein Konstanz. http:// www.am-seerhein.net (letzter Zugriff am 25.12.2014).

Seerhein-Netz (2011): Homepage der Bewohner der Stadt am Seerhein Konstanz. http:// www.am-seerhein.net (letzter Zugriff am 25.12.2014).

Seerhein-Netz (2012): Homepage der Bewohner der Stadt am Seerhein Konstanz. http:// www.am-seerhein.net (letzter Zugriff am 25.12.2014).

Seerhein-Netz (o.J.): Homepage der Bewohner der Stadt am Seerhein Konstanz. http:// www.am-seerhein.net (letzter Zugriff am 25.12.2014).

Stadt Konstanz (2014a): Gemeinderatssitzung vom 20.02.2014. Konzept Prävention und Sicherheit. http://www.konstanz.sitzung-online.de/bi/too20.asp?TOLFDNR=1000710 (letzter Zugriff am 29.03.2016).

Stadt Konstanz (2014b): Ergänzungsvorlage zu Konzept Prävention und Sicherheit. http:// www.konstanz.sitzung-online.de/bi/too20.asp?TOLFDNR=1000767\#allrisAE (letzter Zugriff am 29.03.2016).

Stadt Konstanz (2014c): Beschlussvorlage_0164. Antrag Stadtverwaltung. http://www. konstanz.sitzung-online.de/bi/voo20.asp?VOLFDNR=100014 (letzter Zugriff am 29.03.2016).

Städtebau Bodensee/Hegau GmbH (BHS): Die Stadt am Seerhein. Prospekt Hofgarten 2. http://www.bhs-staedtebau.de/downloads/hofgarten2.pdf (letzter Zugriff am 29.03.2016).

Wehrheim, Jan (2012): Die überwachte Stadt. Sicherheit, Segregation und Ausgrenzung. Opladen: Verlag Barbara Budrich. 
